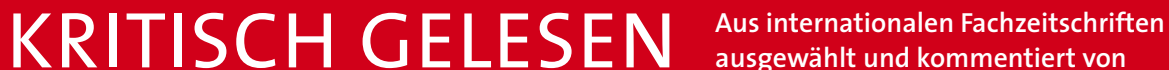

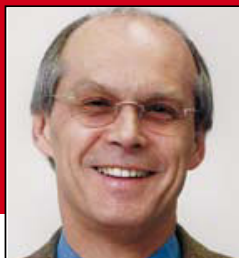

Prof. Dr. med. H. S. Füeß। Isar-AmperKlinikum, KI. MünchenOst, Haar

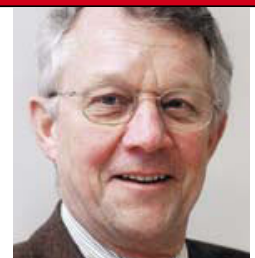

Prof. Dr. med. H. Holzgreve Internist, Kardiologische Praxis, München

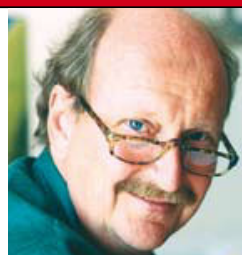

Prof. Dr. med. E. Ernst

Peninsular Medical School, University of Exeter/UK

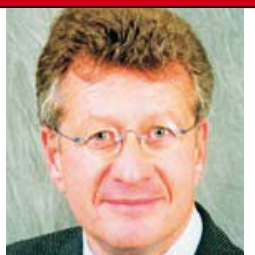

Dr. med. P. Stiefelhagen

Westerwald Krankenhaus, Hachenburg

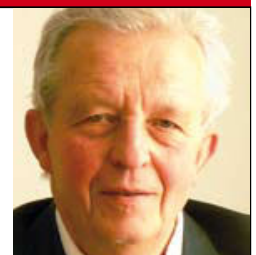

Prof. Dr. med. K. Malberg Immunologie, DresdenLoschwitz

\section{Wer in große Kliniken geht, lebt länger}

Nicht nur für schwierige chirurgische Eingriffe, auch für die Behandlung häufiger Krankheiten wie Herzinsuffizienz und Pneumonie sind die Ergebnisse in Kliniken mit hohen Fallzahlen besser.

- Die Autoren stützen sich auf beeindruckende Zahlen: 734972 stationäre Aufnahmen wegen akuten Myokardinfarkts, 1324287 wegen Herzinsuffizienz und 1418252 wegen Pneumonie in 4128, 4679 bzw. 4673 Kliniken. Anhand von Medicare-Daten wird die Mortalität innerhalb von 30 Tagen in unterschiedlich großen Kliniken der USA überprüft.

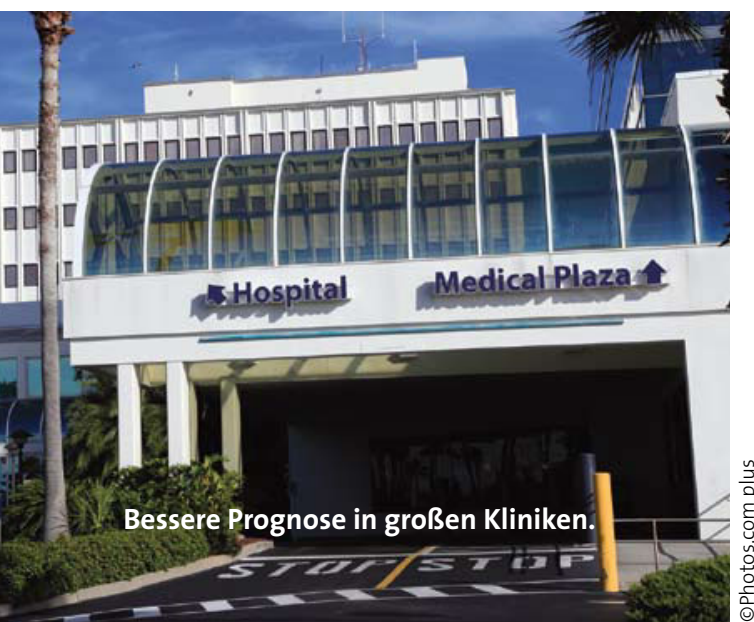

Es besteht zwar eine Heterogenität zwischen den Kliniken, doch als Grundaussage gilt für alle drei Erkrankungen: Je höher die Fallzahlen der Kliniken, desto geringer ist die Mortalität innerhalb von 30 Tagen $(p<0,001)$. Die Abhängigkeit war im Bereich kleiner Fallzahlen so stark ausgeprägt, dass die Mortalitätsrate zwischen etwa 10 und 100 Fällen pro Jahr auf ein Viertel zurückging. Bei höheren Fallzahlen schwächte sich der Zusammenhang zwischen Patientenzahl und Mortalität kontinuierlich ab. Wenn die Korrelation in Einzelschritten von 100 Fällen geprüft wurde, ging die Signifikanz für Myokardinfarkte ab 610 Fällen, für Herzinsuffizienz ab 500 und für Pneunonie ab 210 Patienten verloren, d.h. oberhalb dieser Fallzahlen ist die Mortalitätsrate nicht mehr von der Fallzahl abhängig.

- J. S. Ross et al.

Hospital volume and 30-day mortality for three common medical conditions. New Engl. J. Med. 362 (2010) 1110-1118

\section{Kommentar}

Schon seit vielen Jahren wird der Zusammenhang zwischen der Zahl behandelter Patienten - in Kliniken und von einzelnen Ärzten - und dem Behandlungserfolg anhand von Kriterien wie Aufenthaltsdauer in der Klinik, Komplikationen, Rehospitalisierungen und Mortalität untersucht. Bei technisch und handwerklich schwierigen Eingriffen gilt grundsätzlich: Wo viel gemacht wird, werden die Ergebnisse mit Zunahme von operativer Erfahrung und manueller Geschicklichkeit schließlich besser (,Lernkurve“).

Wenn jetzt gleiche Ergebnisse für Krankheiten wie Herzinsuffizienz und Pneumonie aufgezeigt werden, müssen andere Kriterien die Korrelation zwischen Fallzahlen und Behandlungserfolg beeinflussen. Die Klärung der Ursachen ist schwierig: Große Kliniken haben möglicherweise auch mehr Spezialisten, mehr Intensivbetten und mehr personelle und technische Ressourcen. Auch die Komponente Arzt spielt ein Rolle: Wir haben schon darüber berichtet, dass sehr geschickte und erfahrene Ärzte als Einzelkämpfer in Kliniken mit wenigen Patienten hervorragende Ergebnisse erzielen können (MMW 11/og). Die Grundfrage bleibt: Soll man bei der Zukunftsplanung flächendeckend viele Kliniken mit kleinen Fallzahlen oder wohnsitzferne Zentren mit großen Patientenzahlen propagieren?

H. HolzGreve . 